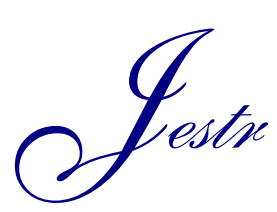

Journal of Engineering Science and Technology Review 10 (5) (2017) 104 - 107

\title{
Location Management Cost Optimization using Normalized Percentile Dwell Time
}

\author{
Swati Swayamsiddha*, Prateek and Sudhansu Sekhar Singh \\ School of Electronics Engineering, KIIT University, Bhubaneswar, Odisha, India
}

Received 2 June 2017; Accepted 16 October 2017

\begin{abstract}
This work proposes Normalized Percentile Dwell Time Distribution to be incorporated into various bio-inspired algorithms such as Genetic Algorithm and Binary Particle Swarm Optimization for optimizing the total cost of Location Management. Dwell time parameter is critically dependent on users' behaviour within the network. The innate advantage of Normalized Percentile dwell time over the conventionally used absolute dwell time is that it can take only fixed values depending on the number of cells per network area. This helps tremendously in reducing the computational overhead and can be applied to any shape of the network as long as the total number of cells per network remains constant. Evidently, from the simulation result the proposed technique works well for the reference networks in terms of scaling and cost reduction.
\end{abstract}

Keywords: Telecommunication computing, Dwell time, Location Management, Genetic Algorithm, Binary Particle Swarm Optimization.

\section{Introduction}

Dwell time is a random variable that describes the amount of time that a mobile can maintain a satisfactory two-way communication link, or simply that a mobile remains in a cell. It is also known as Cell-Residence Time or Mobile Sojourn Time [1]. Dwell time may be fed to the network on a

- Per-user basis,

- Per-cell basis,

- $\quad$ Time of the day based [2].

Dwell time may be broadly classified as shown below:

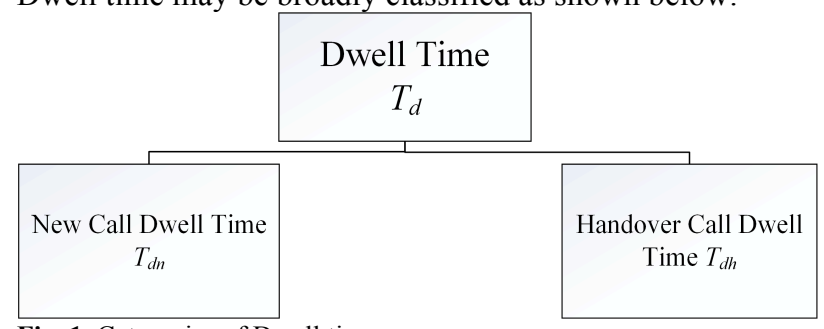

Fig. 1. Categories of Dwell time

New Call Dwell time is the time spent in the cell in which the call originates, whereas Handover Call Dwell Time [3] is the time spent in the cell to which the call gets handed off. The sum total of these two dwell times gives rise to Session Time, which is the entire duration of the call that may cover multiple cells. In the literature, several successful attempts have been taken to model the Dwell time distribution [4].

Bio-inspired meta-heuristic Algorithms may prove to be

*E-mail address: swati.swayamsiddha@gmail.com ISSN: 1791-2377 @ 2017 Eastern Macedonia and Thrace Institute of Technology. All rights reserved. doi:10.25103/jestr.105.13 revolutionary in terms of finding a suitable approach to the location management [5] (L.M.) planning problem. A novel approach would be to integrate the Dwell time parameter into the L.M. cost equation to give a new perspective in hopes of obtaining an improvement in optimization speed and/or efficiency by employing Genetic Algorithm (GA) [6] and Binary Particle Swarm Optimization [7-9] (BPSO), the two-popular bio-algorithms, respectively.

The paper organization is as follows. In the section 2 , the shortfall of the existing dwell time parameter has been briefly mentioned, followed by the introduction of the proposed Normalized Percentile Dwell Time Distribution (NPDT). Section 3 elaborates on the concept of calculation of NPDT parameter and how NPDT may be applied to a mobile location management (MLM) problem [10]. Section 4 provides the simulation results of four different network sizes by incorporating NPDT into GA and BPSO for L.M. cost optimization followed by discussion. Finally, the conclusion and future work is outlined in section 5 .

\section{Problem Formulation}

Cost function shall comprise of update cost and paging cost [11]. Here, a factor of Percentile Dwell Time is proposed. Percentile represents the percentage of data which is below certain threshold. This method of calculation differs from percentage values in the sense that percentage values need to be absolute whereas percentile settles for the relative ranking based on performance of other networks (or) the same network at different times of the day (or) the other users sharing the same network.

So, when a person's mobile handset tries to perform Location update, then instead of using Dwelling Time within the current location area (L.A.) $\left(T_{L . A .}\right)$ as a parameter, the proposed "Percentile Dwell Time" $\left(T_{p d}\right)$ shall be used, which is the percentage of dwell time below the dwell time in that cell. 
Every radio transception involves power and hence cost. The importance of Location Management [12] lies in reducing cost and load on the network by optimizing the cost function. The cost function as accepted by the most researchers is given by

$$
\text { Cost }=\beta \times N_{L U}+N_{P}
$$

Where $N_{L U}$ is the total number of location updates, $N_{P}$ is the total number of paging performed and $\beta$ is a constant to weigh the relative cost of L.U compared to paging [13].

Total cost of L.M is the sum of L.U and Paging cost. Thus, equation (1) may be elaborated as:

$$
\text { Cost }=\beta \times \sum_{i \in R} w_{m i}+\sum_{j=0}^{N} w_{c j} \times v(j)
$$

The term $w_{m i}$ points to the movement weight, whereas $w_{c j}$ points towards call arrival weight. $\mathrm{v}(\mathrm{j})$ is the vicinity factor. Paging cost is simply call rate $(\lambda)$ multiplied by the product of the number of cells and the cost per paging message. L.U cost is the cost per L.U divided by dwelling time within the current L.A.

The advantage of $T_{p d}$ based L.U over traditional L.A based L.U is that in traditional L.A based L.U, an L.U is triggered simply upon boundary crossing from one L.A to another. This may lead to unnecessary L.U cost if the user constantly hops from one L.A to another (assuming that the user is moving along the L.A boundary). But in $T_{p d}$ based L.U, only specific cells are assigned as R.C depending on the user's movement pattern. This avoids excessive L.U cost by triggering a L.U only when the user enters an R.C.Therefore, it is expected that unnecessary location updates shall be minimized, yielding lower mobility management cost [14].

\section{Application of Proposed NPDT to MLM problem}

a) For a network having $\mathrm{N}$ number of cells, where $\mathrm{N}=\mathrm{m} \times \mathrm{n}$, each cell has a particular absolute dwell time value associated per user, averaged over a period of time (usually a complete day).

b) The percentile dwell time $T_{p d}$ is calculated for each cell of the network per user. For example, $T_{p d A}$ for cell A (having absolute dwell time $T_{d A}$ ) is given by

$T_{p d A}=\frac{\text { Number of cells in the network having } T_{d} \text { less than } T_{d A}}{\text { Total number of cells in the network }} \times 100$

c) To take into account the movement pattern [2] of the user, $T_{p d A}$ is divided by a movement factor $M_{i j}$, i $\in \mathrm{fm} \&$ $\mathrm{j} \in \mathrm{n}$, to obtain Normalized percentile dwell time $T_{p d n}$ per cell per user (NPDT).

$$
T_{p d n}=\frac{\text { Number of cells in the network having } T_{d} \text { less than } T_{d A}}{\text { Total number of cells in the network } \times \text { Movement Factor } M_{i j}} \times 100
$$

d) The L.U cost shall be calculated as:

$$
\operatorname{Cost}_{L . U}=\sum_{i \in R} w_{m i}=\frac{\text { Cost per L.U }}{\text { normalized percentile dwell time }}
$$

In Eq. (5) above, the major parameter to be identified is that the denominator of the right-hand side of the equation has Normalized Percentile Dwell Time instead of the conventionally used (Absolute) dwell time.

The cost per call arrival shall be calculated as:

$$
\text { Cost per call arrival }=\frac{\text { Cost }}{\sum_{j=0}^{N} w_{c j}}
$$

Analogy of the concept is shown in Table 1.

Table 1. Dwell time concept tabulation

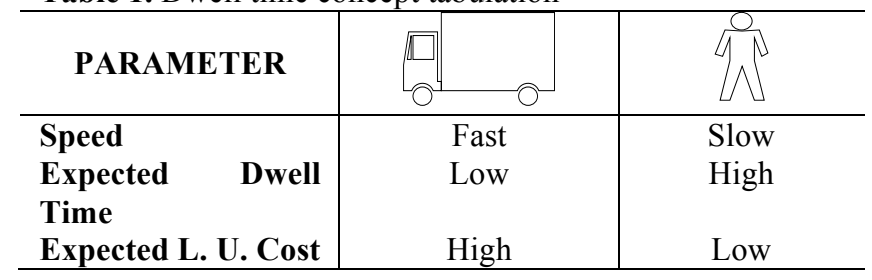

Assumptions:

a) Random Dwell Time Distribution has been assumed since it's the simplest to simulate, and provides a foundation for further studies.

b) The L.A. concept is not to be considered here. Instead, the total number of cells in the entire network is considered as the denominator for percentile calculation.

c) The range of absolute dwell time values is taken as random values between 100 and 3000 [11].

d) Each percentile dwell time value must be divided by a "Movement Factor", which ranges from 0.1 to 50 [2]. The higher the movement factor, the more mobile is the user.

\section{Simulation Results and Discussion}

Simulations were run for symmetric networks using G.A and B.P.S.O, with the varying combinations of the following parameters:

Table 2. Parameters considered for simulations

\begin{tabular}{c|c}
\hline Parameter & Values Considered \\
\hline Network Size & $4 \times 4,6 \times 6,8 \times 8,10 \times 10$ (ref. \\
network data) \\
Population size & 100,120 \\
Number of iterations & $200,250,500$ \\
Absolute Dwell Time & $100-3000$ \\
Distribution & $0.1-50$ \\
Movement Factor & \\
\hline
\end{tabular}

- The Percentile Dwell Time Distribution was integrated at the denominator of the Location update cost,

- The Movement Factor was integrated in the numerator of the Location Update cost.

The results of the simulation are plotted below:

The observations made from the simulation results are as follows:

a) For smaller networks $(4 \times 4)$, BPSO converges up to 16 times faster than GA. But this convergence advantage gets reversed for larger networks (Fig.4).

b) BPSO results in higher level of optimization than GA (Fig. 5). BPSO posts a cost efficiency of $20 \%$ for $4 \times 4,51 \%$ for $6 \times 6,42 \%$ for $8 \times 8$, and $60 \%$ for $10 \times 10$ network over GA. This may be attributed to the additional comparison between the personal best versus the global best that BPSO offers over GA, after each iteration.

c) The cost per call arrival increases with the increase in network size (Fig. 5). This is a common trend for 
both the algorithms, which may be attributed to the larger computational load that is imposed by a larger network consisting of more number of cells, meaning that it becomes less and less effective in locating and paging a mobile user residing in the network.

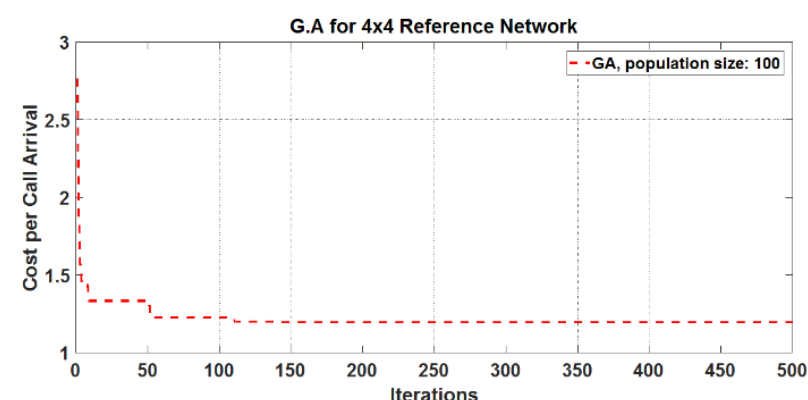

(a.)

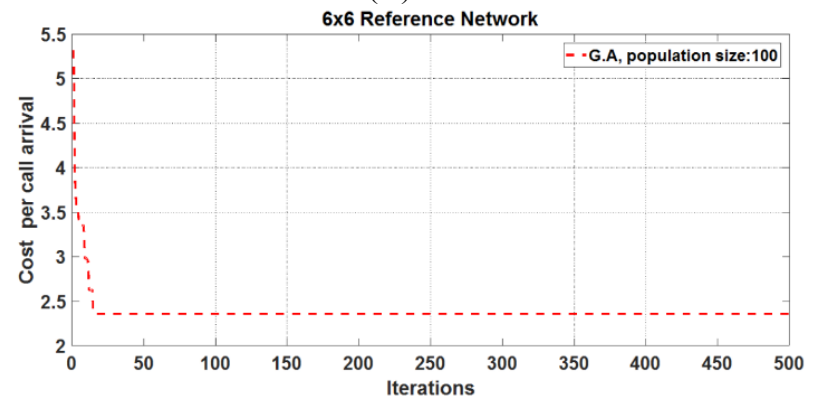

(b.)

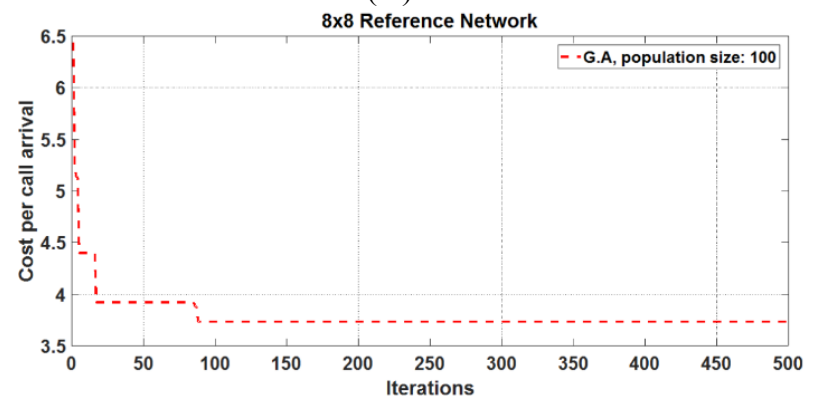

(c.)

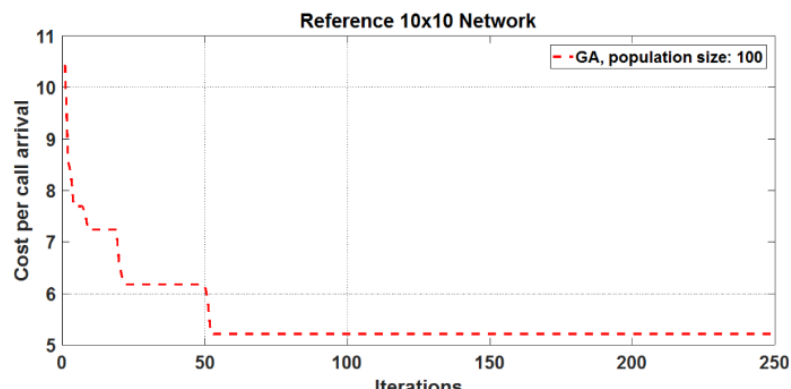

(d.)

Fig. 2. Simulation using Genetic Algorithm for reference networks of

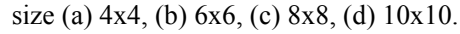

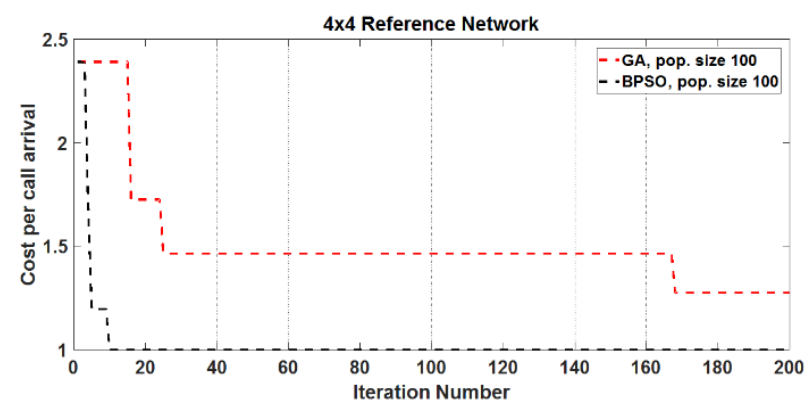

(a.)

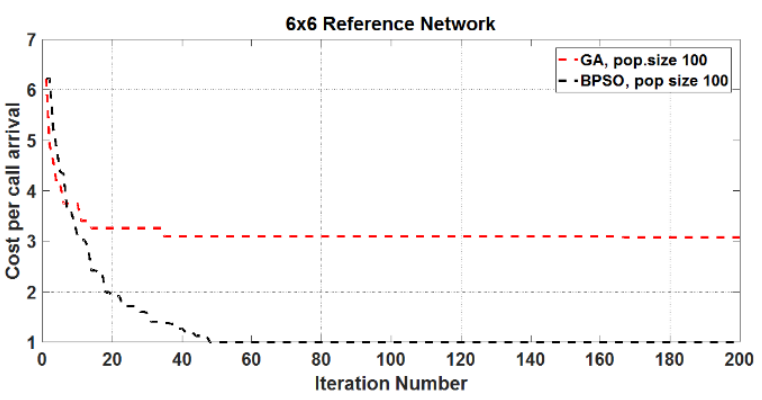

(b.)

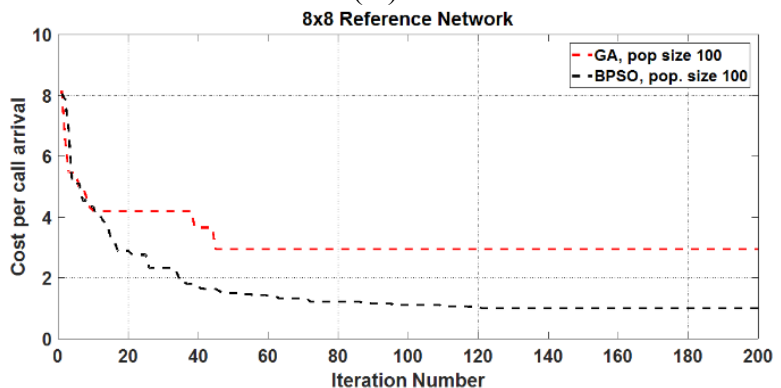

(c.)

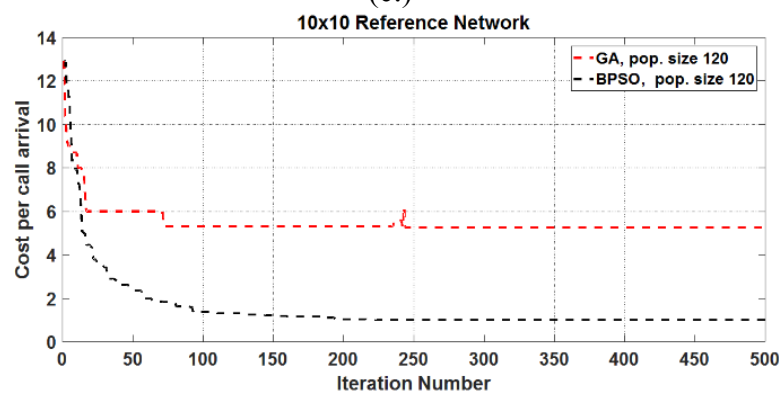

(d.)

Fig. 3. Comparison of Genetic Algorithm and Binary Particle Swarm Optimization for reference networks of size (a) 4x4, (b) $6 \times 6$, (c) $8 \times 8$, (d) $10 \times 10$

d) BPSO exhibits far superior scaling, as compared to GA. This means, when implementing the same algorithm, the percentage increase in the cost per call arrival is lower by up to $70 \%$ when going from $4 \times 4$ to $6 \times 6,46 \%$ when going from $4 \times 4$ to $8 \times 8$, and $67.5 \%$ when going from $4 \times 4$ to $10 \times 10$ network for BPSO as compared to GA.

\section{Conclusion}

The proposed Normalized Percentile Dwell Time Distribution was incorporated as a parameter into the two bio-inspired algorithms, namely, G.A. and B.P.S.O, to optimize location management cost in cellular networks. Based on the proposed method, simulation results were presented for four reference networks of different sizes. The performance of G.A was then compared to B.P.S.O in terms of rate of convergence, optimal cost per call arrival, and scalability with different network sizes. It was observed that the relatively simpler percentile dwell time could indeed be a useful parameter that could boost the performance of the existing networks. Evidently, bio-inspired algorithms provide an interesting way to optimize the total cost, and may be investigated further using combinatorial algorithms too. In the future, the proposed parameter may be implemented to other intelligent algorithms to optimize the total cost of location management. 


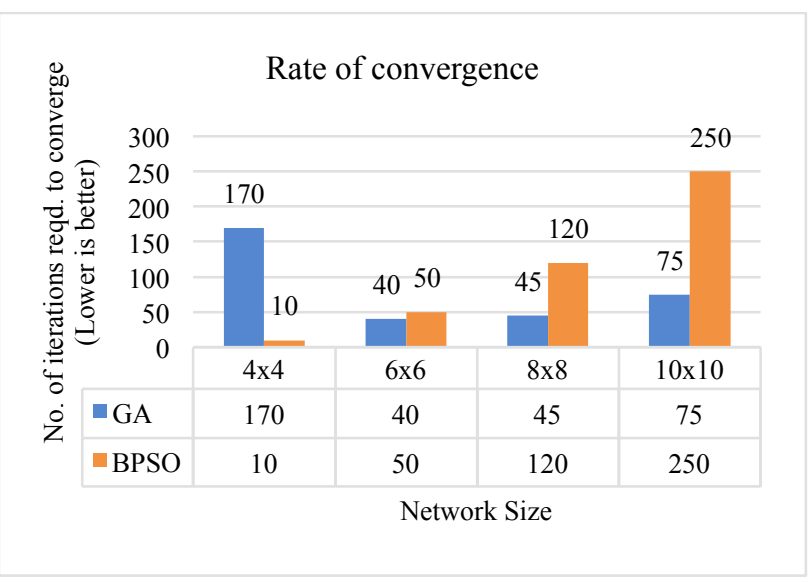

Fig. 4. Comparison w.r.t Rate of Convergence

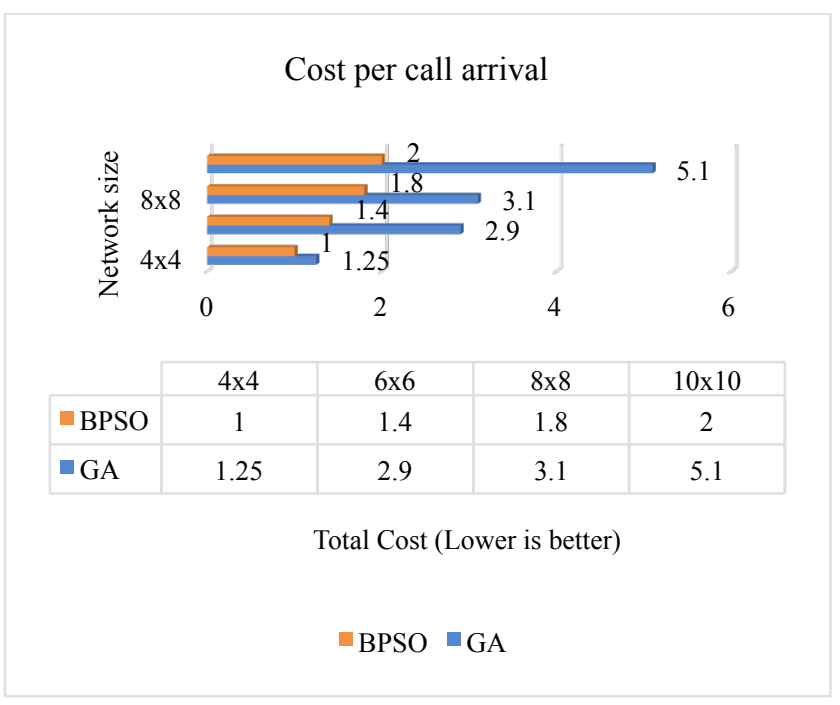

Fig. 5. Comparison w.r.t Cost per call arrival

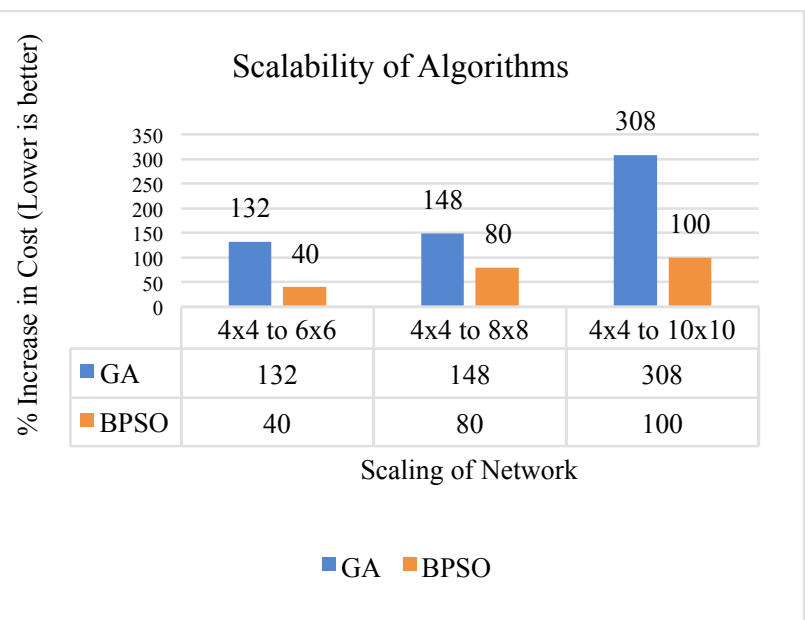

Fig. 6. Comparison w.r.t Scalability of Algorithms

Access article distributed under the terms of the Creative Commons Attribution License

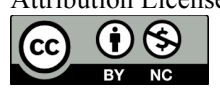

\section{References}

1. Holger Boche and Enrico Jugl, "Dwell time modeling for wireless communication systems and problems of the velocity distribution", European Transactions on Telecommunications, 13(3), pp. 269-278, 2002.

2. James Cowling, "Dynamic Location Management in Heterogeneous Cellular Networks", MIT Thesis. Retrieved November 2004 from http://people.csail.mit.edu/cowling/thesis/jcowling-dynamicNov04.pdf

3. Yan Zhang and Boon-Hee Soong, "Handoff dwell time distribution effect on mobile network performance", IEEE Transactions on Vehicular Technology, 54(4), pp. 1500-08, 2005.

4. Xian Wang and Pingzhi Fan, "Channel holding time in wireless cellular communications with general distributed session time and dwell time", IEEE Communications Letters, 11(2), pp. 158-60, 2007.

5. Babak D. Beheshti, "Review of location management in cellular networks", IEEE Long Island Systems, Applications and Technology Conference, pp. 1-6, 2007.Karen Q. Tian and Donald C. Cox, Mobility Management in Wireless Networks: Data Replication Strategies and Applications, Springer Science \& Business Media, 2007.

6. J. Taheri and A.Y. Zomaya, "A genetic algorithm for finding optimal location area configurations for mobility management", Proceedings of the IEEE Conference on Local Computer Networks 30th Anniversary, Washington, DC, USA, pp. 568-577, 2005.
7. J. Kennedy and R. Eberhart, "Particle swarm optimization", 1995 Proceedings of IEEE International Conference on Neural Networks, 4, pp. 1942- 1948, 1995.

8. S.-S. Kim, G. Kim, J.-H. Byeon and J. Taheri, "Particle Swarm Optimization for Location Mobility Management", International Journal of Innovative Computing, Information and Control, 8, pp. 8387-8398, (ERA Rank: B, IF (2010):1.667), 2012.

9. A. Stacey, M. Jancic and I. Grundy, "Particle swarm optimization with mutation", evolutionary computation, CEC'03. The 2003 Congress, pp. 1425-1430, 2003.

10. Anirudha Chandra and Poulami Das, "Location management in cellular mobile networks", IEEE Potentials magazine, pp. 37-44, 2014.

11. S. R. Parija, P. Addanki, P. K. Sahu and S. S. Singh, "Cost reduction in reporting cell planning configuration using soft computing algorithm", Proceedings of the 3rd International Conference on Frontiers of Intelligent Computing: Theory and Applications (FICTA) 2014. Advances in Intelligent Systems and Computing, 327, 2015.

12. Anwesha Mukherjee and Debashis De, "Location management in mobile networks: a survey", Computer Science Review 19, pp. 114, 2016.

13. S. R. Parija, P. K. Sahu and S. S. Singh, "Cost reduction in location management using reporting cell planning and particle swarm optimization", Wireless Personal Communications, doi:10.1007/s11277-017-4259-3, pp. 1-21, 2017.

14. Keqin Li, "Analysis of distance based location management in wireless communication networks", IEEE Transactions on Parallel and Distributed Systems, 24(2), pp. 225-38, 2013. 